\title{
Cyclin G2 Suppresses Glomerulosclerosis by Regulating Canonical Wnt Signalling
}

\author{
Chenyang Zhao, Jinlan Gao, Sen Li, Qi Liu, Xiaoyu Hou, Shenghuan Liu, Xuesha Xing, \\ Manni Sun, Shusen Wang, and Yang Luo
}

The Research Center for Medical Genomics, Key Laboratory of Cell Biology, Ministry of Public Health, Key Laboratory of Medical Cell Biology, Ministry of Education, College of Basic Medical Science, China Medical University, Shenyang, Liaoning, China

Correspondence should be addressed to Yang Luo; yluo@cmu.edu.cn

Received 3 July 2018; Accepted 30 September 2018; Published 21 October 2018

Academic Editor: Bo Zuo

Copyright (c) 2018 Chenyang Zhao et al. This is an open access article distributed under the Creative Commons Attribution License, which permits unrestricted use, distribution, and reproduction in any medium, provided the original work is properly cited.

Recent data has shown that cyclin G2 (CCNG2) is an atypical cyclin that inhibits cell cycle progression and is often dysregulated in human cancers. The involvement of cyclin G2 in the occurrence and development of diabetic nephropathy (DN) has not been determined. In the present study, we conducted cyclin G2 knockout studies to determine whether this protein regulates glomerulosclerosis in DN mice. We found that cyclin G2 regulated the expression of renal glomerulosclerosis-related proteins via the canonical Wnt signalling pathway in glomerular mesangial cells. A cyclin G2 deficiency resulted in more severe renal injury in $\mathrm{DN}$ mice. These findings provided new insight into the pathogenesis of $\mathrm{DN}$, revealing that cyclin $\mathrm{G} 2$ has a protective role in glomerulosclerosis and is a potential new target for the prevention and treatment of DN.

\section{Introduction}

Diabetic nephropathy (DN) is a relatively common chronic microvascular complication of diabetes that accounts for 30 to $40 \%$ of the morbidities of diabetic patients [1]. DN is also a leading cause of death in these patients [2]. The pathological features of DN include renal glomerular hypertrophy, thickening of the glomerular and tubular cell membranes and the basilar membrane, extracellular matrix accumulation, and, eventually, tubular interstitial fibrosis and glomerulosclerosis. The clinical manifestation of DN involves progressive, irreversible renal dysfunction and ultimately renal failure $[3,4]$.

Several cytokines and signalling pathways participate in the development of DN. The results from numerous studies have demonstrated that the TGF- $\beta /$ Smad signalling pathway, a well-known profibrotic pathway in renal injury, plays a key role in the progression of DN. The PI3K/Akt, p38 MAPK, and JAK/STAT signalling pathways are also involved in the occurrence and development of DN $[5,6]$. However, the pathogenesis of DN is not fully understood. Effective approaches for the treatment and prevention of
DN are lacking. Thus, studies that address the molecular pathogenesis of DN are vital. Evidence suggests that the canonical Wnt signalling pathway participates in several processes involved in renal injury and glomerulosclerosis $[3,7]$. Therefore, the canonical Wnt signalling may have a key role in the occurrence and development of DN.

The canonical Wnt signalling pathway is highly conserved and essential for foetal development, cell fate decisions, and tumour occurrence. Wnt signalling also controls physiological and pathological processes, such as angiogenesis, inflammatory reactions, and glomerulosclerosis [8]. The downstream proteins associated with this pathway are upregulated in the kidneys of patients with diabetes and diabetic mouse models $[9,10]$. Similarly, high-glucose induction activates the canonical Wnt signalling pathway in glomerular podocytes and mesangial cells and causes excessive apoptosis of intrinsic renal cells $[9,11]$. Suppression of this pathway improves proteinuria and renal fibrosis in patients with type 1 diabetes [12]. Recent findings have demonstrated that cyclin G2 has potent tumour-suppressive activity in epithelial ovarian cancer by inhibiting EMT through the attenuation of Wnt signalling [13]. The results from our preliminary studies suggested that 
cyclin G2 (CCNG2) could inhibit canonical Wnt signalling and regulate bone metabolism [14]. However, no evidence has shown whether cyclin G2 specifically functions in the occurrence and development of DN via this pathway.

The expression of cyclin G2 increases in cell cyclearrested and terminally differentiated cells [15]. A large body of evidence indicates that cyclin G2 is an important contributor to suppress the development of cancer; the expression of cyclin G2 is downregulated in thyroid, oral, and breast cancer $[16,17]$. In contrast, cyclin G2 overexpression in colonic cancer cells can induce p53-dependent G1/S-phase cell cycle arrest [15]. The biologic functions of cyclin G2 are mediated through its binding to protein phosphatase 2A (PP2A) and peroxisome proliferator-activated receptor $\gamma(\operatorname{PPAR} \gamma)[18$, 19]. Recent findings suggest that cyclin G2 expression in the fatty tissues of obese patients is associated with steadystate carbohydrate metabolism [20]. However, in patients with type 2 diabetes, the levels of cyclin G2 are positively correlated with that of insulin-degrading enzyme (IDE) [20]. Moreover, insulin and insulin-like growth factor-1 (IGF-1) can significantly downregulate the expression of cyclin G2, thus stimulating DNA synthesis and promoting cell proliferation [21]. Taken together, these data suggest that cyclin G2 may be involved in the pathological processes of diabetes.

In this study, we investigated the regulatory role of cyclin $\mathrm{G} 2$ in the pathological processes of $\mathrm{DN}$ in vitro and in vivo. We found that cyclin G2 contributes to suppressing the occurrence and development of glomerulosclerosis in DN by regulating canonical Wnt signalling. Our results highlight a novel role for cyclin $\mathrm{G} 2$ as a potential target for amelioration of metabolic disease progression.

\section{Materials and Methods}

2.1. Cell Culture, Transfection, and Treatment. The human glomerular mesangial cell line (HMC) was purchased from ATCC (Manassas, VA) and cultured in DMEM containing $10 \%$ foetal bovine serum (Gibco, Life Technologies, Rochester, NY). Cells were transduced with lentivirus particles harbouring CCNG2 or control GFP lentivirus (Genechem, Shanghai, China) and divided into low-glucose (LG; $5.5 \mathrm{mM}$ D-glucose and $24.5 \mathrm{mM}$ L-glucose) and highglucose (HG; $30 \mathrm{mM}$ D-glucose) treatment groups. For serum starvation, cells were grown in DMEM containing 1\% serum for $24 \mathrm{~h}$. For experiments involving the activation of canonical Wnt signalling, cells were treated with CHIR99021 (1 mM; B\&D Systems, Minneapolis, MI) or DMSO control in DMEM for $72 \mathrm{~h}$.

2.2. Western Blot Analysis. We evaluated the regulatory effects of cyclin G2 on the canonical Wnt signalling pathway and on the expression of proteins related to renal injury. Protein concentrations were detected with BCA (Life Technologies). Primary antibodies were anti-collagen IV, anti-cyclin D1, anti- $\beta$-tubulin, anti-phospho- $\beta$-catenin (Ser33/37/Thr41) (Cell Signalling Technology, Boston, MA), anti-GSK3 $\beta$, anti-phospho-GSK3 $\beta$ (Ser9) (Santa Cruz Biotechnology), anti- $\beta$-catenin (Sigma-Aldrich), anti-fibronectin (FN), anti-MMP7, and anti-cyclin G2
(Proteintech, Chicago, IL). All experiments were performed in triplicate.

2.3. Mouse Model of Streptozotocin-Induced Diabetes. To investigate the in vivo functions of cyclin G2, we constructed Ccng $2^{-/-}$mice (Shanghai Model Organisms Center, China) and induced diabetes by injecting streptozotocin (STZ) into Ccng $2^{-/-}$and wild-type (WT; C57BL/6) mice. We assessed the effects of cyclin G2 knockout on the development of $\mathrm{DN}$ and blood glucose and urine protein content. In brief, male WT and Ccng2 $2^{-/-}$mice were housed in cages, fed with normal chow, and maintained on a 12-hour light-dark cycle. Diabetes was induced as described previously [22]. Eight-week-old mice received five consecutive intravenous injections of STZ (50 mg/kg; Sigma-Aldrich) in citrate buffer ( $\mathrm{pH} 4.6$ ) or citrate buffer only. A blood glucose level of $>11.2$ $\mathrm{mmol} / \mathrm{L}$ was confirmed three days after STZ administration at three different time points. Mice were euthanised 16 weeks after STZ injection, and renal tissues were collected. All institutional and national guidelines for the care and use of laboratory animals were followed. All animal experiments were approved by the Animal Care and Use Committee of the Department of Animal Resources, China Medical University.

2.4. Morphological Studies. Renal tissue from $\mathrm{Ccng} 2^{-/-}$mice was examined 16 weeks after the induction of diabetes to determine the effects of cyclin G2 on renal injury and glomerulosclerosis. Specifically, renal tissue was fixed in $4 \%$ paraformaldehyde and embedded in paraffin. Sections $(4 \mu \mathrm{m})$ were examined using periodic acid-Schiff (PAS) and haematoxylin-eosin (HE) stains (Solarbio Life Sciences, Beijing, China). Fifteen randomly selected glomeruli were analysed per animal to evaluate glomerular size. The area of the glomerular tuft and the mesangial matrix index (MMI) were measured using Adobe Photoshop CS6. The MMI was defined as the PAS-positive area in the glomerular tuft region and was calculated from the following equation: $\mathrm{MMI}=$ (PAS-positive area)/(tuft area). The results are presented as the mean \pm standard deviation (SD).

For immunohistochemistry, sections were deparaffinized, rehydrated, and autoclaved for $10 \mathrm{~min}$ in citrate buffer for antigen retrieval. Nonspecific binding was blocked by incubation for $30 \mathrm{~min}$ in $10 \%$ goat or rabbit serum. Samples were incubated with primary antibody (anti- $\beta$-catenin, anticollagen IV, or anti-FN) at $4^{\circ} \mathrm{C}$ overnight. After washing in PBS, the sections were incubated with secondary antibody, and specific staining was visualised using the Ultrasensitive S-P Kit (streptavidin-peroxidase; Sigma-Aldrich).

2.5. Statistical Analysis. Data are presented as the mean \pm SD. Statistical analysis was performed using Student's $t$-test for comparisons between two groups or with the paired $t$ test for comparison between more than two groups. Statistical significance was defined as $P<0.05$.

\section{Results}

3.1. Cyclin G2 Inhibits the Expression of Tubular Glomerulosclerosis-Related Proteins in HMC Cells. To evaluate the 


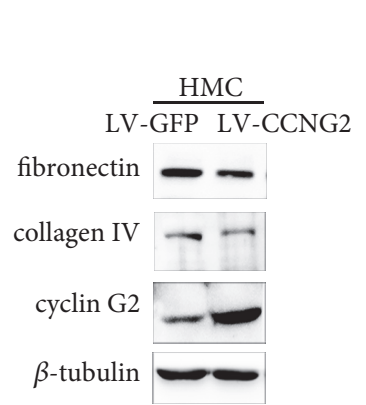

(a)

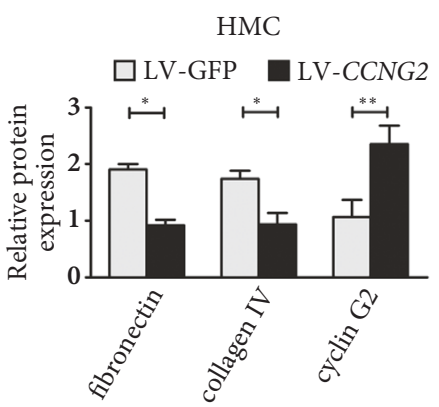

(b)

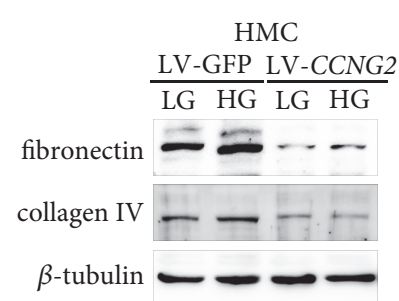

(c)

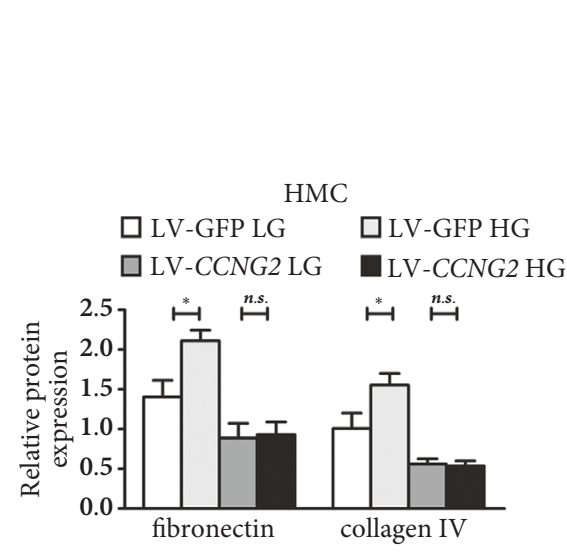

(d)

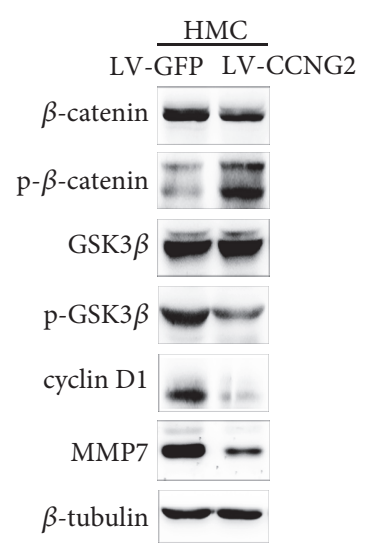

(e)

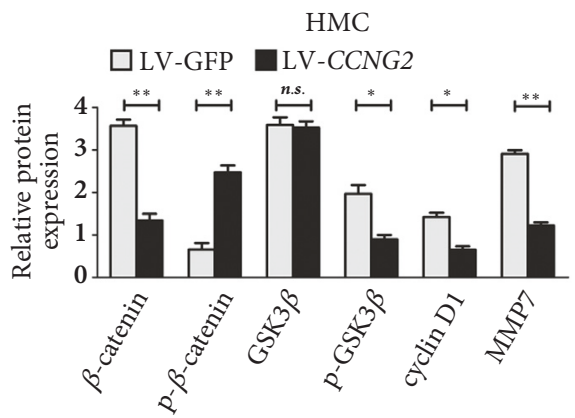

(f)

HMC

$\frac{\text { LV-GFP }}{\text { LG HG }} \frac{\text { LV-CCNG2 }}{\text { LG HG }}$

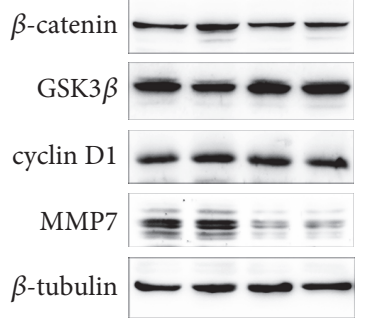

(g)

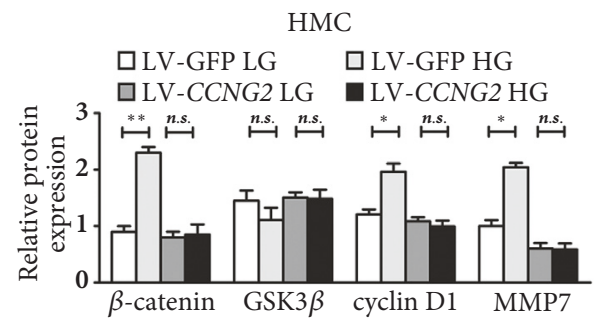

(h)

FIGURE 1: Cyclin G2 inhibits the expression of glomerulosclerosis-related proteins and canonical Wnt signalling in HMC cells. (a) Representative western blots and (b) densitometry for FN and collagen IV in HMC cells transduced with lentiviral particles harbouring CCNG2 (LV-CCNG2) or GFP (LV-GFP; control group). (c) Representative western blots and (d) densitometry for FN and collagen IV in CCNG2-overexpressing HMC cells exposed to $30 \mathrm{mM}$ D-glucose (HG) or $5.5 \mathrm{mM}$ D-glucose + $24.5 \mathrm{mM}$ L-glucose (LG; control group) for $72 \mathrm{~h}$. (e) Representative western blots and (f) densitometry for $\beta$-catenin, $\mathrm{p}$ - $\beta$-catenin, GSK3 $\beta$, p-GSK3 $\beta$, cyclin D1, and MMP7 in HMC transduced with lentiviral particles expressing CCNG2 (LV-CCNG2) or GFP (LV-GFP; control group). (g) Representative western blots and (h) densitometry for $\beta$-catenin, GSK3 $\beta$, cyclin D1, and MMP7 in CCNG2-overexpressing HMC cells exposed to HG or LG for $72 \mathrm{~h}$. Values are presented as the mean $\pm \mathrm{SD}(\mathrm{n}=3) ;{ }^{*} p<0.05,{ }^{* *} p<0.01$, n.s.: not significant.

effect of cyclin G2 on glomerulosclerosis, we overexpressed cyclin G2 in HMC cells using lentiviral (LV-CCNG2) transduction and analysed the expression of glomerulosclerosisrelated proteins. Compared to the control group (LV-GFP [i.e., green fluorescent protein]), the levels of FN and collagen IV were significantly downregulated in cyclin G2-overexpressing HMC cells (Figures 1(a) and 1(b)).

High-glucose levels in diabetes may accelerate renal injury in DN [23]. To evaluate whether cyclin G2 inhibited the expression of glomerulosclerosis-related proteins under high-glucose conditions, we cultured HMC cells in a high concentration of glucose for $72 \mathrm{~h}$, which led to the upregulation of FN and collagen IV. Overexpression of cyclin G2 suppressed this high-glucose-induced upregulation (Figures $1(\mathrm{c})$ and $1(\mathrm{~d})$ ). These results demonstrated an antiglomerulosclerosis activity for cyclin G2 in the context of diabetes in HMC cells.

3.2. Cyclin G2 Suppresses the Canonical Wnt Signalling Pathway in HMC Cells. Ectopic expression of cyclin G2 in 


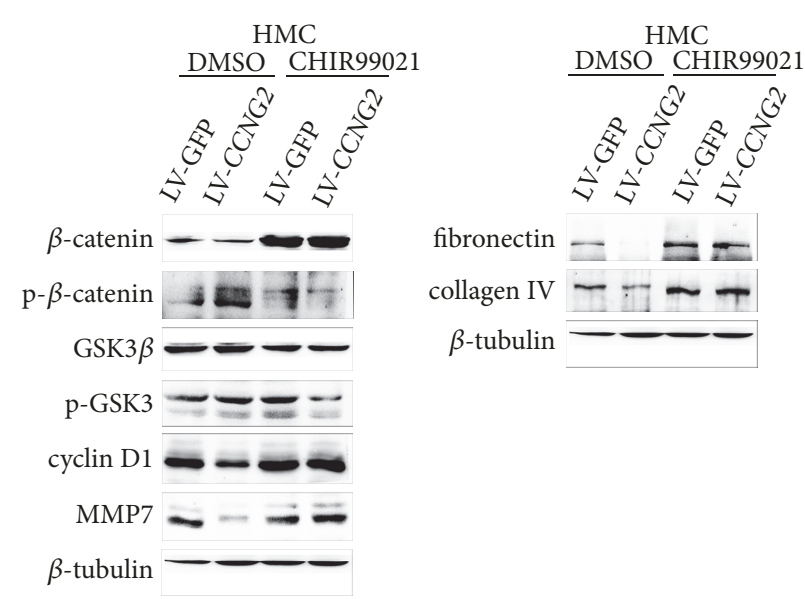

(a)

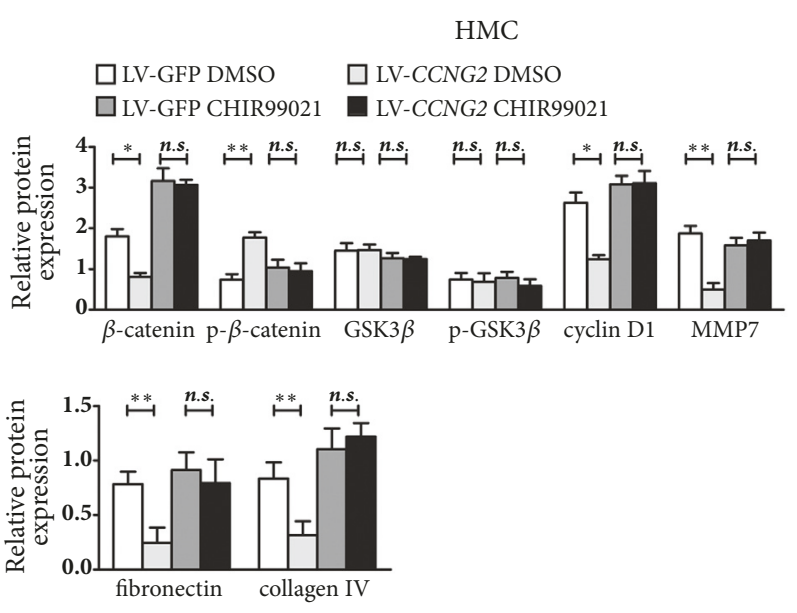

(b)

FIGURE 2: Cyclin G2 inhibits the expression of proteins associated with glomerulosclerosis via canonical Wnt signalling. (a) Representative western blots and (b) densitometry for $\beta$-catenin, p- $\beta$-catenin, GSK3 $\beta$, p-GSK3 $\beta$, cyclin D1, MMP7, FN, and collagen IV in cyclin G2-overexpressing HMC cells (LV-CCNG2) treated with $5 \mu \mathrm{M}$ CHIR99021 or DMSO (control group) for $72 \mathrm{~h}$. Values are presented as the mean $\pm \mathrm{SD}(\mathrm{n}=3) ;{ }^{*} p<0.05,{ }^{* *} p<0.01, n . s$. not significant.

HMC cells inhibited the expression of $\beta$-catenin and its targets, cyclin D1 and MMP7. Cyclin G2 overexpression also upregulated the levels of phosphorylated (p)- $\beta$-catenin (Ser33/37/Thr41) and downregulated p-GSK3 $\beta$ (Ser9) levels (Figures 1(e) and 1(f)). When HMC cells were cultured in high glucose, the levels of $\beta$-catenin, cyclin D1, and MMP7 were induced. However, overexpression of cyclin G2 abolished this effect (Figures $1(\mathrm{~g})$ and $1(\mathrm{~h})$ ). Therefore, cyclin G2 could suppress the activation of the canonical Wnt signalling pathway by high glucose in HMC cells.

\subsection{Cyclin G2 Alters the Expression of Glomerulosclerosis-} Related Proteins by Regulating Canonical Wnt Signalling. To determine the regulatory mechanisms for cyclin G2 in renal glomerulosclerosis, we activated the canonical Wnt signalling pathway using the GSK3 $\beta$ inhibitor CHIR99021 [24]. In the absence of CHIR99021, cyclin G2 overexpression inhibited the expression of proteins associated with canonical Wnt signalling and glomerulosclerosis. CHIR99021 treatment abolished the effect of cyclin G2 overexpression on Wnt signalling and decreased its effect on FN and collagen IV levels (Figures 2(a) and 2(b)). These data suggested that cyclin G2 regulates the expression of glomerulosclerosisrelated proteins via canonical Wnt signalling in HMC cells.

\subsection{Cyclin G2 Deficiency Results in More Severe Renal Injury} in DN Mice. Renal morphology and the severity of glomerulosclerosis in $\mathrm{Ccng}^{-/-}$and WT DN mice were evaluated by PAS and HE staining. Our results revealed that the glomerular basement membrane index was significantly increased in $\mathrm{Ccng}^{-1-}$ DN mice compared to WT DN mice (Figures 3(a), 3(b), and 3(c)). In these DN renal tissues, the already elevated levels of FN and collagen IV were further increased following cyclin G2 knockout (Figures 3(d) and $3(\mathrm{e})$ ). Increased collagen IV expression in the DN mouse kidney following cyclin G2 knockout was confirmed by immunohistochemistry (Figure 3(f)). Therefore, cyclin G2 appeared to ameliorate renal injury in a diabetes mouse model.

3.5. Cyclin G2 Deficiency Activates Canonical Wht Signalling in the Kidneys of DN Mice. We evaluated renal tissues of Ccng $2^{-/-}$and WT mice by immunohistochemistry and western blotting. We found that the levels of $\beta$-catenin, cyclin D1, and MMP7 in the renal cortex of $\mathrm{Ccng}^{-/-}$mice were higher than the levels in the renal cortex of WT mice (Figures 4(a) and 4(b)). These findings confirmed the regulatory effect of cyclin G2 on the components of the canonical Wnt signalling pathway in vivo.

To evaluate the effects of cyclin G2 inhibition on canonical Wnt signalling in diabetes, we assessed protein expression levels of Wnt signalling factors in the renal tissues of $C \mathrm{cng} 2^{-/-}$ and WT DN mice. We found that the levels of $\beta$-catenin, cyclin D1, and MMP7 in Ccng $2^{-/-}$DN mice were substantially higher than those of WT DN mice (Figures 4(c)-4(e)). Therefore, a lack of cyclin G2 resulted in enhanced canonical Wnt signalling in renal tissues of DN mice.

\section{Discussion}

DN has a complex pathogenesis that includes maladaptive metabolic mechanisms induced by high glucose and renal injury caused by an interaction of environmental and genetic factors $[3,25]$. Involvement of cyclin G2 in the process of glomerulosclerosis in diabetes has not been reported previously. Other investigators have noted that cyclin G2 expression is negatively correlated with glucose and human insulin tolerance and regulates the metabolism of fatty tissues in internal organs [20]. Several authors have suggested that cyclin G2 interacts with PPAR $\gamma$ to regulate lipogenesis [19]. In this study, we demonstrated that cyclin G2 knockout caused increased glomerular hypertrophy, accumulation of 

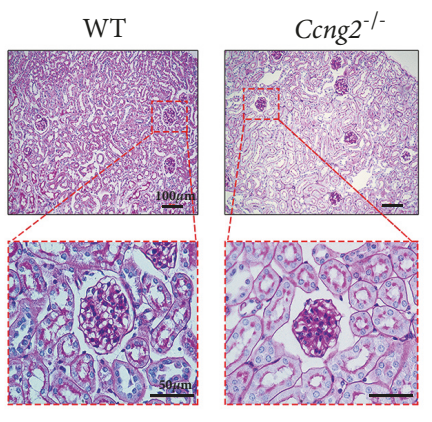

(a)
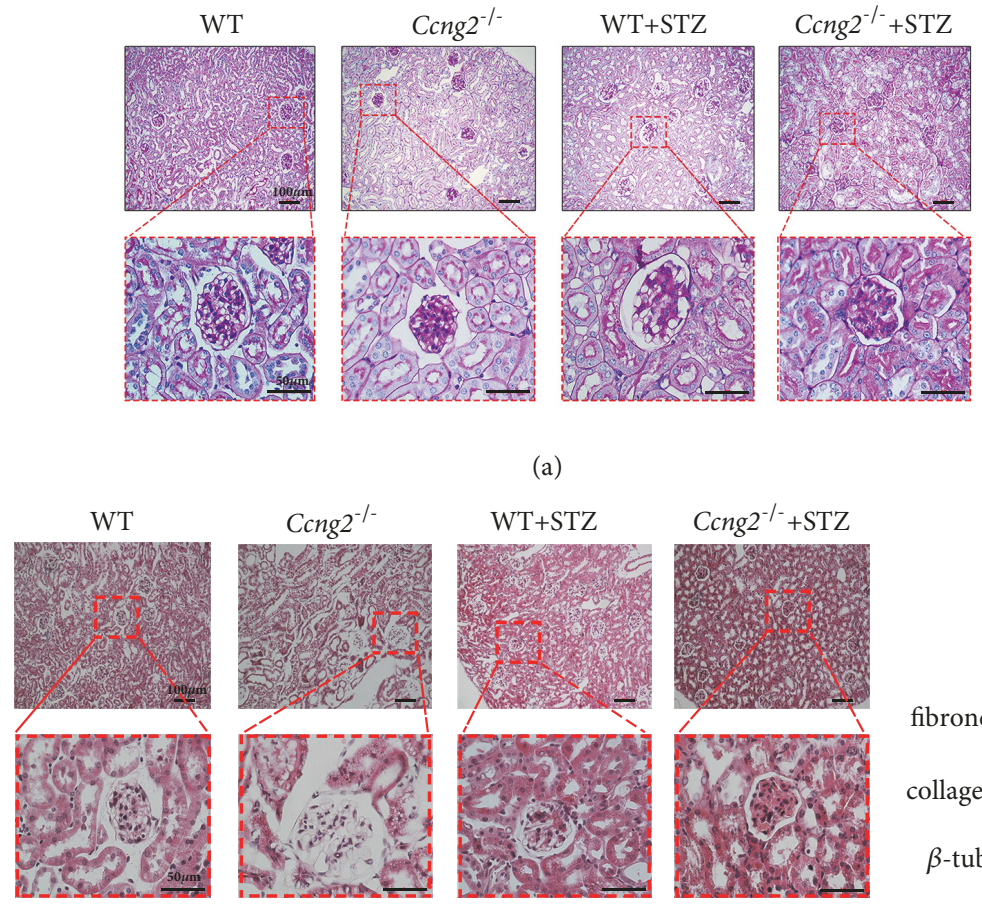

(c)

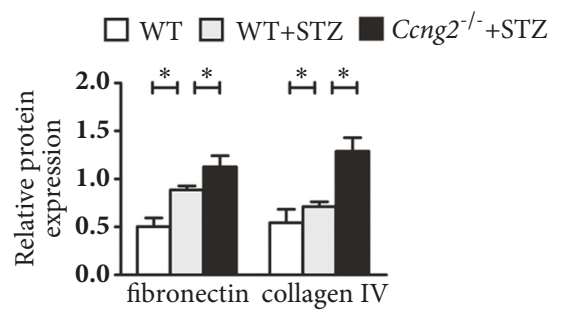

(e)
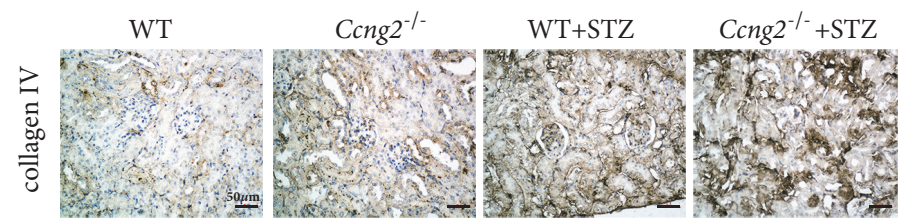

(f)

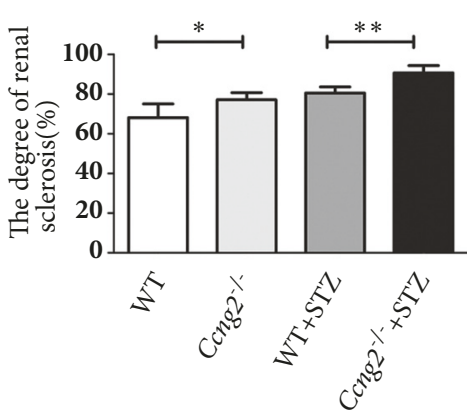

(b)

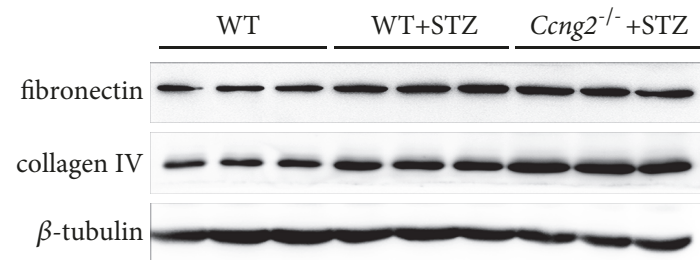

(d)

FIGURE 3: Cyclin G2 deficiency increases renal injury in DN mice. (a, b) PAS staining depicting the degree of renal sclerosis in control WT and $C c n g 2^{-/-}$mice and STZ-induced WT and Ccng $2^{-/-}$DN mice (original magnification, 100×, 400×). (c) HE staining shows glomerular structures (original magnification, $100 \times, 400 \times$ ). (d) Representative western blots and (e) densitometry for FN and collagen IV in the renal cortex of control WT and STZ-induced WT and Ccng $2^{-1-}$ DN mice. (f) Representative collagen IV immunohistochemistry for control WT and Ccng $2^{-/-}$mice and STZ-induced WT and Ccng $2^{-/-}$DN mice (original magnification, 200×). Values are presented as the mean \pm SD (n $=3) ;{ }^{*} p<0.05,{ }^{* *} p<0.01$, n.s.: not significant.

mesangial matrix, tuberous sclerosis, and other pathophysiological changes. Hence, cyclin G2 may be involved in the occurrence and development of DN.

Tubular mesangial cells control the glomerular filtration rate and provide capillary support. Notably, the accumulation of the mesangial matrix and formation of a mesangial extracellular matrix are essential features of DN [26]. By overexpressing cyclin G2 in HMC cells, we found that cyclin G2 significantly inhibited the expression of glomerulosclerosisrelated proteins. Conversely, cyclin G2 knockout increased expression of glomerulosclerosis-related proteins in the mouse kidney.

Effective control of blood glucose may be sufficient to reduce the risks of diabetic complications. High-glucose treatment of HMC cells significantly upregulated the levels of glomerulosclerosis-related proteins. However, these highglucose effects were suppressed by cyclin G2 overexpression. We observed increases in the content of accumulated mesangial matrix in Cong $2^{-/-}$DN mice compared to WT DN mice. Glomerulosclerosis-related proteins were also elevated, and other pathophysiologic changes were evident. These results revealed a novel function of cyclin G2 as an inhibitor of renal injury progression in $\mathrm{DN}$.

Canonical Wnt signalling is an evolutionarily conserved signal transduction pathway, which affects cellular events that modulate various disease processes and plays a critical role in kidney development [27]. High-glucose conditions and oxidative stress promote the activation of canonical Wnt signalling in the kidneys in diabetic animal models [12]. Investigators have demonstrated that the Huang 


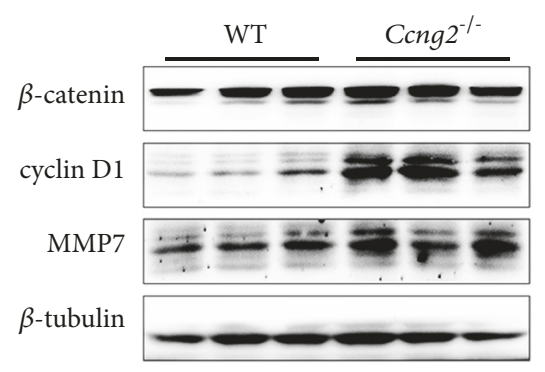

(a)

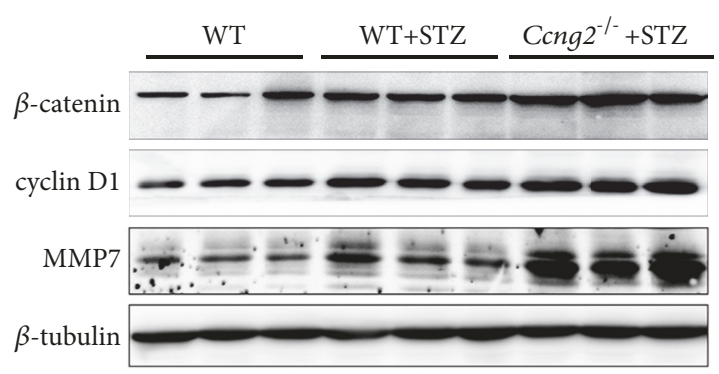

(c)

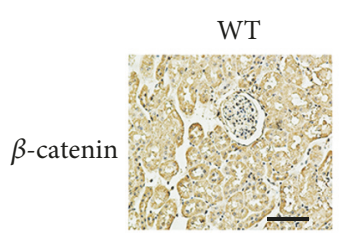

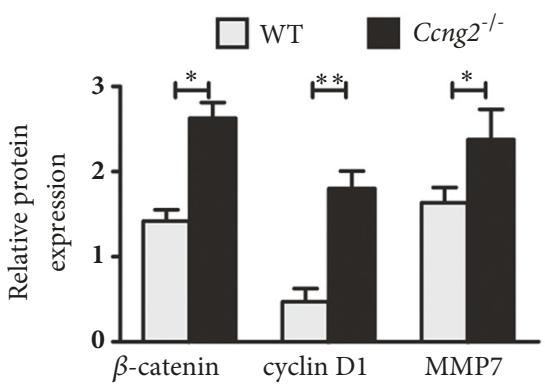

(b)

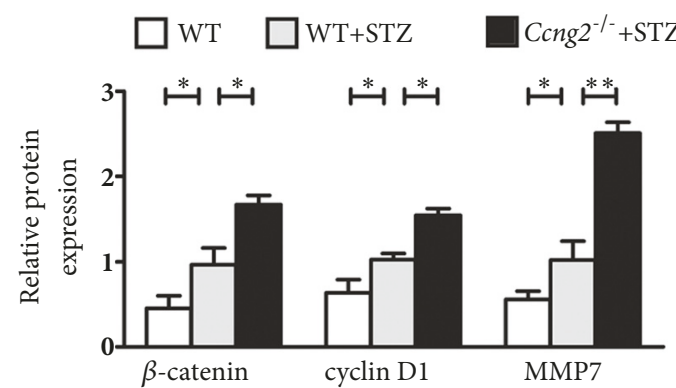

(d)
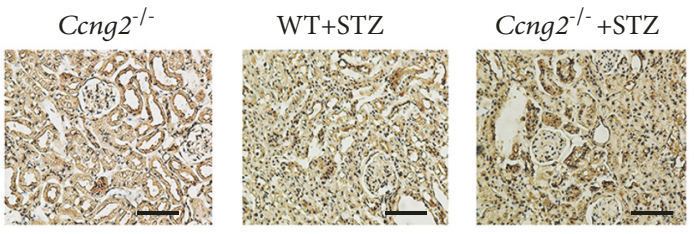

(e)

FIGURE 4: A lack of cyclin G2 promotes the activation of canonical Wnt signalling in a DN mouse model. (a) Representative western blots and (b) densitometry for $\beta$-catenin, cyclin D1, and MMP7 in the renal cortex of WT and Ccng $2^{-/-}$mice. (c) Representative western blots and (d) densitometry for $\beta$-catenin, cyclin D1, and MMP7 in the renal cortex of control WT and STZ-induced WT and Ccng2 ${ }^{-/-}$DN mice. (e) Representative $\beta$-catenin immunohistochemistry for control WT and $C c n g 2^{-/-}$mice and STZ-induced WT and $C c n g 2^{-/-}$DN mice (original magnification, 200×). Values are presented as the mean $\pm \mathrm{SD}(\mathrm{n}=3) ;{ }^{*} p<0.05,{ }^{* *} p<0.01$, n.s.: not significant.

Gan formula improved remnant renal function and alleviated glomerulosclerosis and tubulointerstitial fibrosis potentially by suppressing the canonical Wnt signalling pathway [28]. These results indicated that the canonical Wnt signalling pathway plays a vital role in DN. However, the precise regulatory mechanisms governing this process are unknown.

Recent studies suggested that cyclin G2 could inhibit canonical Wnt signalling in bone metabolism and epithelial ovarian cancer $[13,14]$. In this study, overexpression of cyclin G2 in HMC cells resulted in the negative regulation of proteins involved in canonical Wnt signalling and suppressed the high-glucose-induced upregulation of these proteins. Conversely, cyclin G2 knockout increased the expression of canonical Wnt signalling factors in the mouse kidney and activated the canonical Wnt signalling pathway in the kidneys of DN mice. Therefore, cyclin G2 negatively regulates canonical Wnt signalling both in vitro and in vivo. Furthermore, treatment of HMC cells with the GSK3 $\beta$ inhibitor CHIR99021 (activator of Wnt signalling) abrogated the inhibitory effects of cyclin G2 on canonical Wnt signalling and glomerulosclerosis-related proteins.

\section{Conclusions}

A cyclin G2 deficiency precipitated the pathophysiological changes in mice with DN. Therefore, cyclin G2 is protective against DN progression, specifically against glomerulosclerosis. The regulation of the pathological progression in the $\mathrm{DN}$ mouse kidney by cyclin G2 occurred through its repression of canonical Wnt signalling. Our findings delineate a previously unidentified function of cyclin G2 as a protector in the pathologic progression of glomerulosclerosis in DN. These results provide potential new targets for the prevention and treatment of DN in the clinical setting.

\section{Data Availability}

The data used to support the findings of this study are available from the corresponding author upon request.

\section{Ethical Approval}

All animal experiments were approved by the Institutional Animal Care and Use Committee of China Medical University (Shenyang, China). 


\section{Conflicts of Interest}

The authors declare that they have no competing interests.

\section{Authors' Contributions}

Yang Luo designed the experiments. Chenyang Zhao conducted the main experiments and wrote the paper. Jinlan Gao, Sen Li, and Qi Liu helped with plasmid construction. Xiaoyu Hou, Shenghuan Liu, and Xuesha Xing maintained the mice and performed genotyping. Manni Sun and Shusen Wang performed PAS and Masson staining.

\section{Acknowledgments}

This work was supported by the Foundation of the Education Department of Liaoning Province (nos. LZDK201703 and 21601137) and the National Natural Science Foundation of China (nos. 81400851, 81170543, and 81571440).

\section{References}

[1] L. Yang, L. Wu, Y. Fan, and J. Ma, "Vitamin D receptor gene polymorphisms in association with diabetic nephropathy: A systematic review and meta-analysis," BMC Medical Genetics, vol. 18, no. 1, 2017.

[2] C. Mega, E. Teixeira-De-Lemos, R. Fernandes, and F. Reis, "Renoprotective effects of the dipeptidyl peptidase- 4 inhibitor sitagliptin: a review in type 2 diabetes," Journal of Diabetes Research, vol. 2017, Article ID 5164292, 14 pages, 2017.

[3] L. Xiao, M. Wang, S. Yang, F. Liu, and L. Sun, "A glimpse of the pathogenetic mechanisms of $\mathrm{Wnt} / \beta$-catenin signaling in diabetic nephropathy," BioMed Research International, vol. 2013, Article ID 987064, 7 pages, 2013.

[4] J. R. Mader, Z. T. Resch, G. R. McLean et al., "Mice deficient in PAPP-A show resistance to the development of diabetic nephropathy," Journal of Endocrinology, vol. 219, no. 1, pp. 5158, 2013.

[5] F. C. Brosius III, C. C. Khoury, C. L. Buller, and S. Chen, "Abnormalities in signaling pathways in diabetic nephropathy," Expert Review of Endocrinology \& Metabolism, vol. 5, no. 1, pp. 51-64, 2010.

[6] A. Mima, "Diabetic nephropathy: protective factors and a new therapeutic paradigm," Journal of Diabetes and its Complications, vol. 27, no. 5, pp. 526-530, 2013.

[7] Y. Guo, L. Xiao, L. Sun, and F. Liu, "Wnt/ $\beta$-catenin signaling: a promising new target for fibrosis diseases," Physiological Research, vol. 61, no. 4, pp. 337-346, 2012.

[8] N. Maruotti, A. Corrado, A. Neve, and F. P. Cantatore, "Systemic effects of Wnt signaling," Journal of Cellular Physiology, vol. 228, no. 7, pp. 1428-1432, 2013.

[9] C. Dai, D. B. Stolz, L. P. Kiss, S. P. Monga, L. B. Holzman, and Y. Liu, "Wnt/ $\beta$-catenin signaling promotes podocyte dysfunction and albuminuria," Journal of the American Society of Nephrology, vol. 20, no. 9, pp. 1997-2008, 2009.

[10] T. Kawakami, S. Ren, and J. S. Duffield, "Wnt signalling in kidney diseases: dual roles in renal injury and repair," The Journal of Pathology, vol. 229, no. 2, pp. 221-231, 2013.

[11] C. L. Lin, J. Y. Wang, Y. T. Huang, Y. H. Kuo, K. Surendran, and F. S. Wang, "Wnt/beta-catenin signaling modulates survival of high glucose-stressed mesangial cells," Journal of the American Society of Nephrology, vol. 17, no. 10, pp. 2812-2820, 2006.

[12] T. Zhou, X. He, R. Cheng et al., "Implication of dysregulation of the canonical wingless-type MMTV integration site (WNT) pathway in diabetic nephropathy," Diabetologia, vol. 55, no. 1, pp. 255-266, 2012.

[13] S. Bernaudo, M. Salem, X. Qi et al., "Cyclin G2 inhibits epithelial-to-mesenchymal transition by disrupting Wnt/betacatenin signalling," Oncogene, vol. 35, no. 36, p. 4828, 2016.

[14] J. Gao, Q. Liu, X. Liu et al., "Cyclin G2 Suppresses EstrogenMediated Osteogenesis through Inhibition of Wnt/ $\beta$-Catenin Signaling," PLoS ONE, vol. 9, no. 3, p. e89884, 2014.

[15] M. Zimmermann, A. S. Arachchige-Don, M. S. Donaldson, R. F. Dallapiazza, C. E. Cowan, and M. C. Horne, "Elevated cyclin G2 expression intersects with DNA damage checkpoint signaling and is required for a potent G2/M checkpoint arrest response to doxorubicin," The Journal of Biological Chemistry, vol. 287, no. 27, pp. 22838-22853, 2012.

[16] Y. Kim, S. Shintani, Y. Kohno, R. Zhang, and D. T. Wong, "Cyclin G2 dysregulation in human oral cancer," Cancer Research, vol. 64, no. 24, pp. 8980-8986, 2004.

[17] M. Adorno, M. Cordenonsi, M. Montagner et al., "A Mutantp53/Smad complex opposes p63 to empower TGF $\beta$-induced metastasis," Cell, vol. 137, no. 1, pp. 87-98, 2009.

[18] D. A. Bennin, A. S. Arachchige Don, T. Brake et al., "Cyclin G2 associates with protein phosphatase $2 \mathrm{~A}$ catalytic and regulatory B' subunits in active complexes and induces nuclear aberrations and a G1/S phase cell cycle arrest," The Journal of Biological Chemistry, vol. 277, no. 30, pp. 27449-27467, 2002.

[19] V. Aguilar, J.-S. Annicotte, X. Escote, J. Vendrell, D. Langin, and L. Fajas, "Cyclin G2 regulates adipogenesis through PPAR gamma coactivation," Endocrinology, vol. 151, no. 11, pp. 5247$5254,2010$.

[20] O. Pivovarova, C. Von Loeffelholz, I. Ilkavets et al., "Modulation of insulin degrading enzyme activity and liver cell proliferation," Cell Cycle, vol. 14, no. 14, pp. 2293-2300, 2015.

[21] M. Zimmermann, A. P. S. Arachchige-Don, M. S. Donaldson, T. Patriarchi, and M. C. Horne, "Cyclin G2 promotes cell cycle arrest in breast cancer cells responding to fulvestrant and metformin and correlates with patient survival," Cell Cycle, vol. 15, no. 23, pp. 3278-3295, 2016.

[22] S. Yung, M. K. M. Chau, Q. Zhang, C. Z. Zhang, and T. M. Chan, "Sulodexide decreases albuminuria and regulates matrix protein accumulation in C57BL/6 mice with streptozotocin induced type I diabetic nephropathy," PLoS ONE, vol. 8, no. 1, Article ID e54501, 2013.

[23] X. Wu, Y. Gao, L. Xu et al., "Exosomes from high glucose-treated glomerular endothelial cells trigger the epithelial-mesenchymal transition and dysfunction of podocytes," Scientific Reports, vol. 7, no. 1, 2017.

[24] A. S. Bock, N. D. Leigh, and E. C. Bryda, "Effect of Gsk3 inhibitor CHIR99021 on aneuploidy levels in rat embryonic stem cells," In Vitro Cellular \& Developmental Biology - Animal, vol. 50, no. 6, pp. 572-579, 2014.

[25] Y.-M. Sun, Y. Su, J. Li, and L.-F. Wang, "Recent advances in understanding the biochemical and molecular mechanism of diabetic nephropathy," Biochemical and Biophysical Research Communications, vol. 433, no. 4, pp. 359-361, 2013.

[26] K. V. Lemley, M. Elger, I. Koeppen-Hagemann et al., “The glomerular mesangium: capillary support function and its failure under experimental conditions," The Clinical Investigator, vol. 70, no. 9, pp. 843-856, 1992. 
[27] C. Hu, L. Sun, L. Xiao et al., "Insights into the mechanisms involved in the expression and regulation of extracellular matrix proteins in diabetic nephropathy," Current Medicinal Chemistry, vol. 22, no. 24, pp. 2858-2870, 2015.

[28] L. Mo, X. Xiao, S. Song et al., "Protective effect of Huang Gan formula in 5/6 nephrectomized rats by depressing the Wnt/ $\beta$-catenin signaling pathway," Drug Design, Development and Therapy, vol. 9, pp. 2867-2881, 2015. 


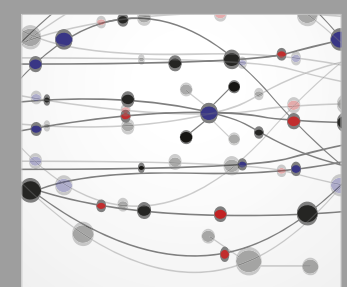

The Scientific World Journal
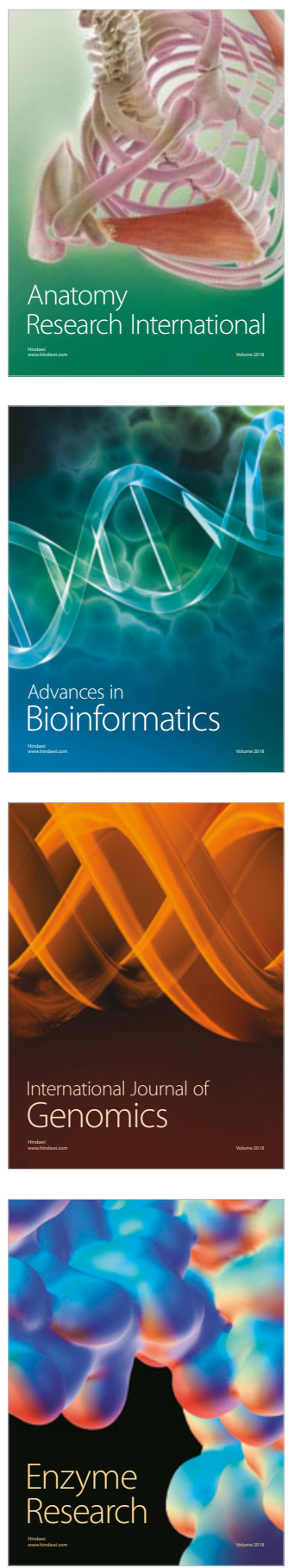
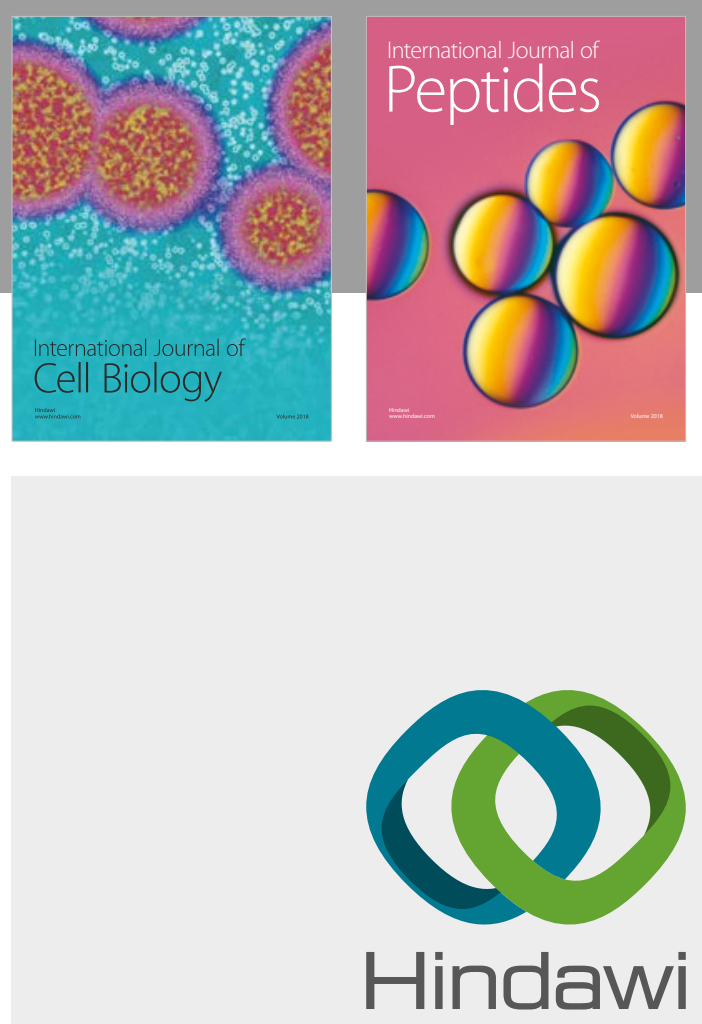

Submit your manuscripts at

www.hindawi.com
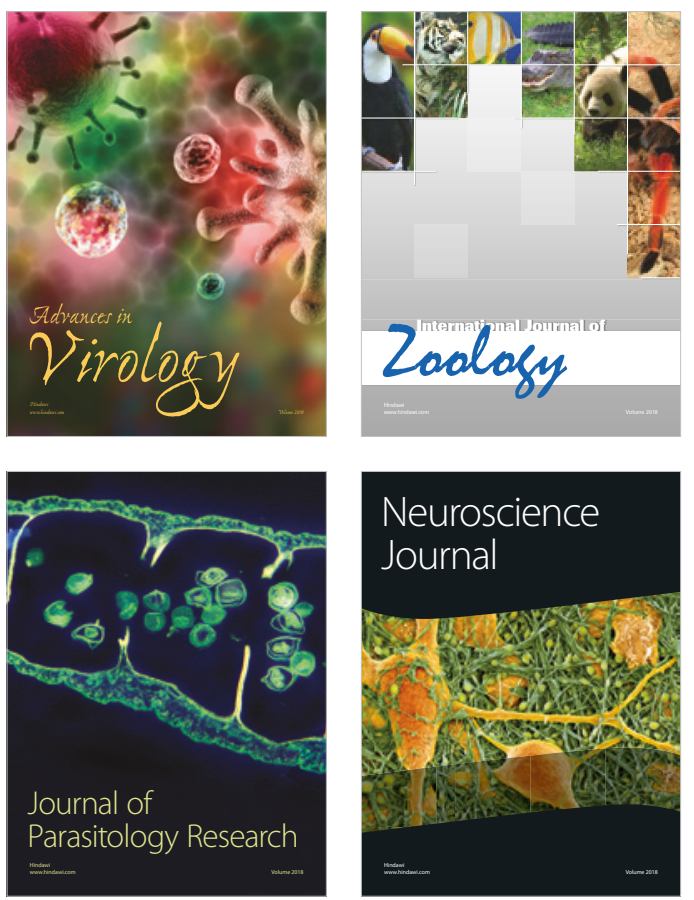
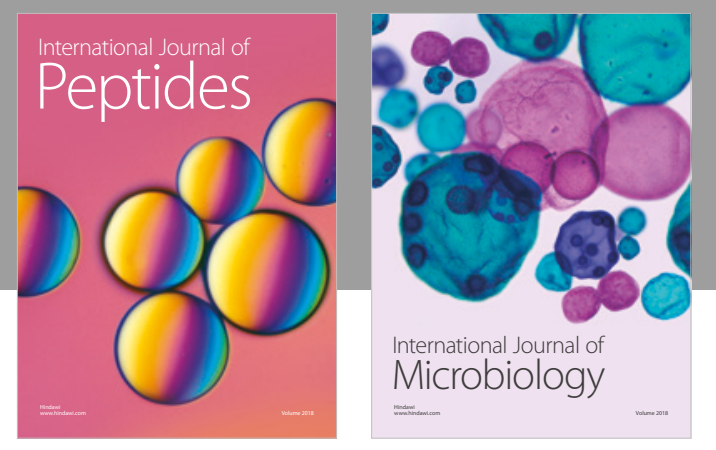

nternational Journal of Microbiology
Journal of
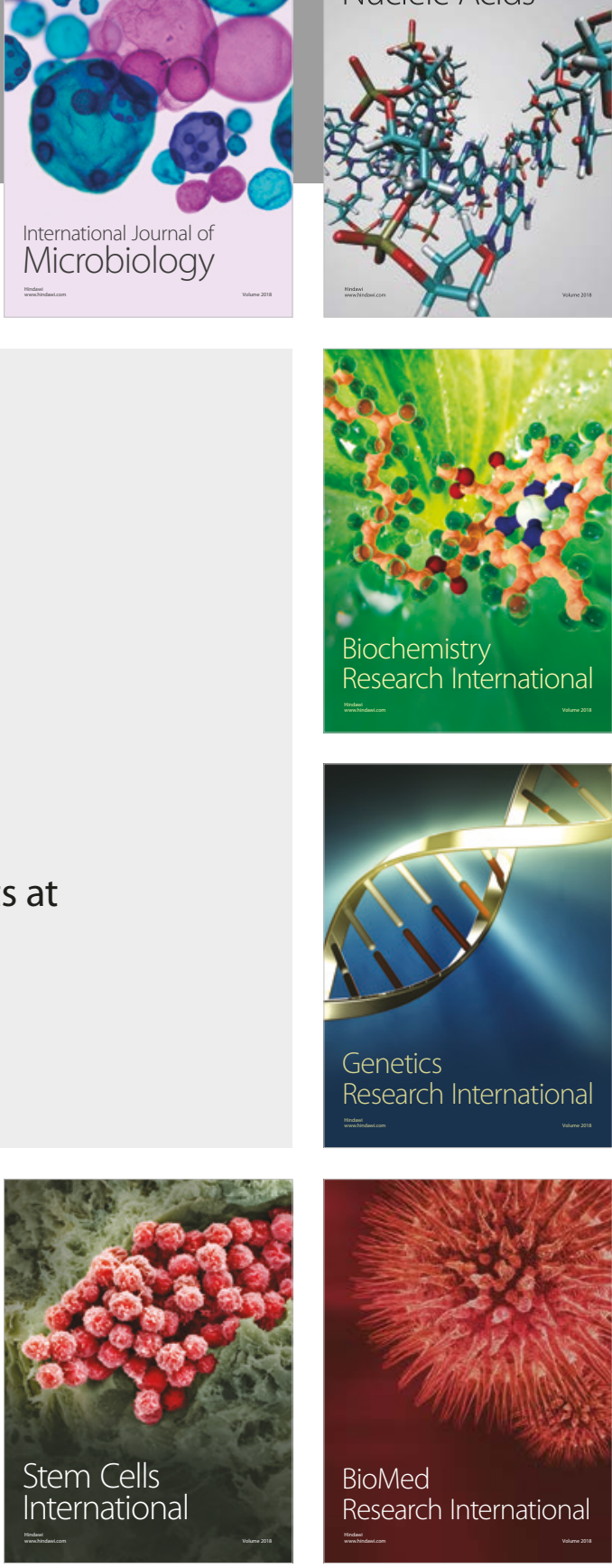
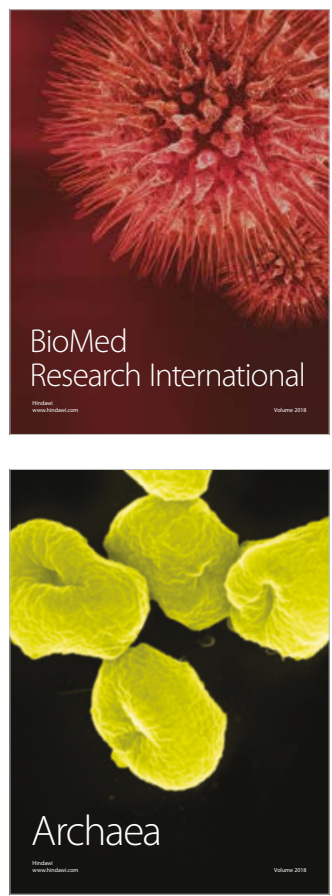